\title{
Symposium:
}

\section{Grundlagen des Maximal- und Schnellkrafttrainings in Freiburg vom 6. bis 8. Oktober 1983}

Veranstalter: Bundesinstitur für Sportwissenschaft Institut für Sport und Sportwissenschaft der Universität Freiburg

Dieses Symposium ist als Kontakt-Veranstaltung für die in diesem Bereich arbeitenden Wissenschaftler und die in den entsprechenden Sportarten tätigen Trainer konzipiert. Es sollen einerseits die jüngsten Forschungsergebnisse aus grundlagen- und anwendungsorientierter Sicht vorgetragen und andererseits die praktizierten Trainingsmethoden im Spitzensport vorgestelit werden.

Diese Gegenüberstellung wird aufzeigen, inwieweit diese Konzepte übereinstimmen oder divergieren. Die Diskussion zwischen Wissenschaftlern und Trainern soll im Vordergrund stehen.

Folgende Themenbereiche sind für Haupt- und Kurzreferate vorgesehen:

Neurophysiologische Grundlagen - Muskelfaserstruktur - Muskelelastizität und reaktives Kraftverhalten - Muskelermüdung und Krafttraining im Kurzzeitbereich (bis $30 \mathrm{sec}$ ).

Dimensionen des Maximalkraft- und Schnellkraftverhaltens, ihre Diagnose und optimalen Trainingsmethoden.

Belastbarkeit und Krafttraining im Kindes- und Jugendalter.

Die Praxis des Krafttrainings wird in folgenden Sportarten und -disziplinen vorgestellt: Gewichtheben - Bodybuilding - Sprint - Hochsprung.

Mit Kurzreferaten (15 Min.) können darüber hinaus Ergebnisse und Perspektiven zum obigen Themenkreis in das Symposium eingebracht werden. Interessierte Kollegen werden gebeten, ihre Bereitschaft zu einem Kurzreferat bis zum 1. April 1983 anzuzeigen. Um die gleichzeitige Obersendung einer ein- bis zweiseitigen Kurzfassung wird gebeten. Die Quartierbeschaffung muß direkt über das Verkehrsamt Freiburg geregelt werden (Verkehrsamt der Stadt Freiburg, Rottedrring 14, 7800 Freiburg i. Br. - Telefon 0761/2 16-32 89). Das genaue Programm des Symposiums wird Anfang Mai veröffentlicht.

Alle Anfragen sind zu richten an das

Institut für Sport und Sportwissenschaft

der Universität Freiburg,

Schwarzwaldstr. 175, 7800 Freiburg 